\title{
USING RELATIVE CAPACITY TO MEASURE HABITAT SUITABILITY
}

\author{
AleXANDRe H. HiRZEL \\ Department of Ecology and Evolution, University of Lausanne, \\ CH-1015 Lausanne, Switzerland
}

\begin{abstract}
Habitat suitability (HS) modeling relates a species' potential presence/absence to a set of environmental variables. Because of stochastic or demographic population fluctuations, relating abundance to environment is difficult and generally requires time-series of population-density data. Here, I propose an approach to compute relative capacity of a set of habitat classes. I defined relative capacity of a habitat class as being proportional to its carrying capacity, and replaced time-series of density measures with spatial replicates. A hypothetical environment was first divided into several habitat classes and population densities were measured in a sample of these habitats. Three methods of computing relative capacity were compared: (1) maximum density per habitat class, (2) coefficients of a Generalized Linear Model (GLM) of the habitat densities, and (3) slopes of isodars computed for all pairs of habitat classes. Accuracy of these methods was evaluated using spatially-explicit demographic simulations. I investigated biological assumptions (species' sensitivity, reactivity and selectivity, regional stochasticity) and sampling design (sample size, distance between pairs of habitat classes, number of habitat classes, uncertainty over abundance). GLM and maximum-density methods provided accurate results when at least five site-pairs were available. Isodars outperformed other methods in landscapes with many habitat classes, but are best suited to large or noise-free data sets. Optimal performance was reached with more than 50 site-pairs and five habitat classes.
\end{abstract}

Keywords: habitat suitability, ideal free distribution, isodar theory, population dynamics, simulated data, spatial ecology, virtual species

\section{INTRODUCTION}

Habitat suitability (HS) modeling relates a species' occurrence to a set of environmental variables to model its ecological niche and predict its potential distribution (Soberón, 2007; Hirzel and Le Lay, 2008). One of the main outputs of these models is the predictive HS map, which can be used to delineate the spatial distribution of a species, to predict potential range of an invader or a disease, to delineate protected areas for an endangered species, or to map biodiversity hotspots (Guisan and Thuiller, 2005; Peter- 
son, 2006). The field of HS modeling has developed quickly during the last 15 years, generating a large number of methods and applications (Guisan and Zimmermann, 2000; Pearce and Boyce, 2006).

But what does HS represent exactly? As the input data are occurrences of a species, i.e., a binary variable, it cannot be a number or density of individuals. While methods based on presence and absence data (e.g., logistic regression) claim to compute "probabilities of occurrence" (Guisan and Zimmermann, 2000), this term refers correctly to the probability of finding the species, given the entire sample. As such, it depends strongly on the ratio between detected presences and absences, which itself depends on the extent of the study area. Thus, rare species have low probabilities of occurrence, even in those places where all of their environmental requirements are fulfilled. Resource Selection Function theory (Manly et al., 2002) more correctly uses the term "relative probability of occurrence". The value computed by HS models should be thought of as a measure of environmental similarity between the focal area and a hypothetical species' optimal habitat. Thus, it is a combination of resource, resting site, and breeding site availability, which directly affects survival, reproduction, colonization, and extinction risk (Jaquiéry et al., 2008).

In some cases, wildlife managers require estimates of population density rather than HS. Although many HS techniques can theoretically predict species' abundances (Guisan and Zimmermann, 2000), it generally proves difficult (Potts and Elith, 2006), especially for animal species (e.g., Olivier and Wotherspoon, 2005). This is because environmental and demographic stochasticity may drive populations far below their carrying capacity. The problem is analogous to the HS vs. presence prediction. Predicting HS is different from predicting presence because the latter involves population dynamics (dispersal, stochastic extinctions, history), which the former can ignore (Soberón and Peterson, 2005; Soberón, 2007; Hirzel and Le Lay, 2008). Similarly, it is easier to predict carrying capacity than abundance.

Carrying capacity cannot be measured with a snapshot survey. Instead, it usually requires a time series of density and growth rate measurements on a fluctuating population (Freckleton et al., 2006). Although possible to obtain on a limited number of sites, such long-term studies are not typically feasible given the large sample sizes required by the usual HS methods. Moreover, time series require several years before providing usable data. Because some ecosystems change rapidly, time is often what we lack most.

I propose an alternative approach based on finding the conversion function from HS to a discrete area's carrying capacity (see also Discussion). This approach replaces time series by a snapshot density survey over a sufficiently large area and provides a rapid way to evaluate potential population sizes. I explore three analytical methods, with controls, and test their sensitivity to various sampling and biological conditions by applying them to simulated virtual species (Hirzel et al., 2001; Austin et al., 2006).

\section{RELATIVE CAPACITY ANALYSES}

The carrying capacity of an area represents the population size that its resources and 
conditions can maintain at equilibrium (Begon et al., 1996). As it depends only on the prevailing environment, it is a function of HS. According to this definition, the area need not be homogeneous; rather, environmental heterogeneity may be a component of HS. The area may also not be clearly delimited and can be any arbitrary partitioning of the landscape (e.g., a grid). In this way, this definition of carrying capacity is well-suited to GIS raster structure.

For my purpose, I define "relative carrying capacity", $K^{\prime}$, as a value directly proportional to carrying capacity, $K$. There is an unknown coefficient $\alpha$ that verifies $K^{\prime}=$ $\alpha K$. By convention, relative capacity is scaled to range from 0 to 1 . Relative capacity facilitates comparisons among quantitatively different environments in a study area (e.g., environment A can harbor a population twice as large as environment B). While the focus here involves computation of $K^{\prime}$, evaluation of the proportionality coefficient $\alpha$ may be conducted by standard time-series analyses.

Relative capacity analyses are based on a HS map and a snapshot density survey of an area large enough for regional stochasticity to occur, where regional stochasticity is the spatially autocorrelated component of environmental stochasticity (Hanski, 1991, 1998): close populations experience similar conditions, while the dynamics of populations located far apart are decoupled. Thus, a sufficiently large study area would have specific regions experiencing various levels of crowding at the same time. As regions may be composed of diverse environmental conditions, it is possible to compare the environment-dependent density at various crowding levels. In other words, spatial replaces temporal variability. The methods then proceed as described below.

For a set of sites, assume that we have habitat suitability values and standardized measurements of population density. The HS values could stem from either a statistical HS model or expert knowledge. Sites are classified to $k$ classes according to environmental conditions. Some of the sites must be sufficiently far apart for their population dynamics to be independent. I propose three methods (and a randomly-selected control sample) to assess the relative capacity of these HS classes, as described below.

The "Maximum" method assumes that, although populations are fluctuating, at least one of each suitability class is, probabilistically, at its highest density. The method consists of recording, for each HS class $s(1 \leq s \leq k)$, the maximum observed density $N_{M, s}$. Then, relative capacities are obtained by $K^{\prime}=N_{M, s} / \max \left(N_{M, s}\right)$.

The second method is based on fitting a Generalized Linear Model (GLM) relating population density, $N$, to habitat suitability, $S$, using the equation: $N=\alpha_{0}+\alpha_{1} S+\alpha_{2} S^{2}$. The fitted model is then used to compute a predicted value $N_{G, s}$ for each HS class $s$. The relative capacities are finally obtained by $K^{\prime}=N_{G, s} / \max \left(N_{G, s}\right)$. This model assumes that there is a linear or quadratic relationship between abundance and HS.

The third method is based on the theory of isodars (Morris, 1988, 2003). This theory assumes that, given the choice among a set of sites differing by their suitability and crowding, individuals select the site(s) that maximizes their fitness (Ideal Free Distribution, IFD, Fretwell and Lucas, 1969; Krivan et al., 2008). The basic assumptions of IFD are that individuals have a global knowledge of the crowding and suitability of all available sites, are free to settle in any site, and there is no dispersal cost. However, 


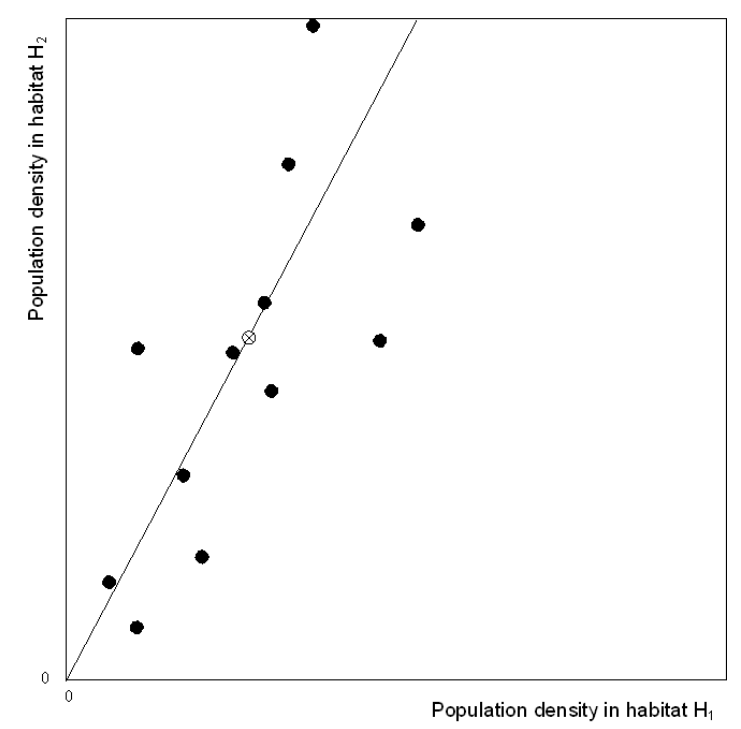

Fig. 1. Isodar plot. Each dot represents densities in pairs of nearby sites, having habitats $\mathrm{H}_{1}$ and $\mathrm{H}_{2}$, respectively, at various levels of crowding. According to the ideal free distribution hypothesis, individuals distribute themselves among the two habitats to equalize average fitness. The slope of the isodar curve (black line), which passes through the data centroid (crossed circle), represents the relative suitability of the two habitats. In this example, the 2:1 slope indicates that habitat $\mathrm{H}_{2}$ is two times more suitable than is habitat $\mathrm{H}_{1}$.

the IFD appears even when those conditions are not completely met (Cressman and Krivan, 2006). As negative density-dependence counterbalances the benefits of habitat suitability, this tends to homogenize the individual average fitness across all close sites (Emlen et al., 2003). The Isodar method requires a set of density measures taken in pairs of neighboring sites that differ by their environment, and at several levels of crowding. Plotting the population densities in the two sites on the same graph reveals how the individuals distribute themselves at various levels of crowding (Fig. 1). If the IFD assumption is verified, the density pairs define a curve of equal fitness (called "isodar", in homage to Darwin). The shape of the isodar reveals the relative suitability of the studied habitats (Fig. 1). Often, the density measurements come from time-series monitored on a few pairs of study sites (e.g., Morris, 1992; Lin and Batzli, 2002; Shochat et al., 2002). In the present case, all densities are measured simultaneously in a large number of paired sites and pairs are chosen to minimize the distance within pairs, while maximizing the distance between pairs (e.g., Knight and Morris, 1996). Thus, the sites within a pair are assumed to experience the same level of crowding, which may differ from the crowding of other pairs. 
Table 1

Example of an isodar slope matrix. Each coefficient is computed as the slope of linear isodar with null intercept, for all combinations of habitats (here, four habitat classes, $\mathrm{H}_{1}$ to $\mathrm{H}_{4}$ ). In this example, $\mathrm{H}_{4}$ is 2 times more suitable than $\mathrm{H}_{3}$, which is 1.5 times more suitable than $\mathrm{H}_{2}$, etc. Because relative suitability is evaluated pairwise, inconsistencies may arise (e.g., $\mathrm{H}_{4}$ may be $2 \times 1.5=3$ times more suitable than $\mathrm{H}_{2}$, or 2.9 times more suitable). These weights are compiled to provide capacities relative to $\mathrm{H}_{1}$. The last row provides the relative capacity of each habitat class, after rescaling to a maximum value of 1

\begin{tabular}{lllll}
\hline & $\mathrm{H}_{1}$ & $\mathrm{H}_{2}$ & $\mathrm{H}_{3}$ & $\mathrm{H}_{4}$ \\
\hline $\mathrm{H}_{1}$ & & 1.2 & 1.7 & 3.5 \\
$\mathrm{H}_{2}$ & & & 1.5 & 2.9 \\
$\mathrm{H}_{3}$ & & & 2.0 \\
Relative capacity & 1.00 & 1.20 & 1.75 & 3.49 \\
Scaled relative capacity & 0.287 & 0.344 & 0.501 & 1.000 \\
\hline
\end{tabular}

The "isodar" method proceeds as follows: (1) For each combination of habitat classes (say, for $k=3: \mathrm{H}_{1}-\mathrm{H}_{2}, \mathrm{H}_{1}-\mathrm{H}_{3}, \mathrm{H}_{2}-\mathrm{H}_{3}$ ), a list is made of geographically-close pairs of sites belonging to this set of classes. (2) for each list, pairwise densities are plotted to fit an isodar model. Preliminary simulations showed that the best results were given by the straight line linking the origin $(0,0)$ to the centroid of the distribution (Fig. 1). (3) This step provides an isodar slope for each habitat class combination, which can be represented within a triangular slope matrix (Table 1). Let $a_{i j}$ be the relative suitability of habitat $\mathrm{H}_{j}$ in reference to habitat $\mathrm{H}_{i}\left(\mathrm{H}_{j}\right.$ is $a_{i j}$ times more suitable than $\left.\mathrm{H}_{i}\right)$. When $k$ is large, some combinations may not be represented; these are ignored for remaining analyses. (4) Integrate the information contained in the slope matrix into relative capacity as follows: Assign to $\mathrm{H}_{1}$ a reference relative capacity of $f\left(\mathrm{H}_{1}\right)=1$. From it, compute the relative capacity of $\mathrm{H}_{2}$ by $f\left(\mathrm{H}_{2}\right)=a_{12} f\left(\mathrm{H}_{1}\right)$. For $\mathrm{H}_{3}$, relative capacity can be computed by $f^{\prime}\left(\mathrm{H}_{3}\right)=a_{13} f\left(\mathrm{H}_{1}\right)$ or $f^{\prime \prime}\left(\mathrm{H}_{3}\right)=a_{23} f\left(\mathrm{H}_{2}\right)$. The two ways usually provide similar but not identical relative capacities. Take the mean of these two values. All remaining relative capacities are computed in a similar way. (5) Finally, the relative capacities are rescaled as in the two previous methods by dividing them by the maximum values. Table 1 provides a numerical example of these computations.

\section{METHODS}

To test and compare the domain of application of these methods, I used a virtual-species approach as it allows for a variety of computer experiments and provides a framework for objectively evaluating results (Hirzel et al., 2001; Austin et al., 2006).

\section{SIMULATING POPULATION DYNAMICS}

The program HEXASPACE (Hirzel, 2001; Hirzel et al., 2007) was used for modeling population dynamics. It consists of a virtual landscape composed of 200-by-200 hexago- 

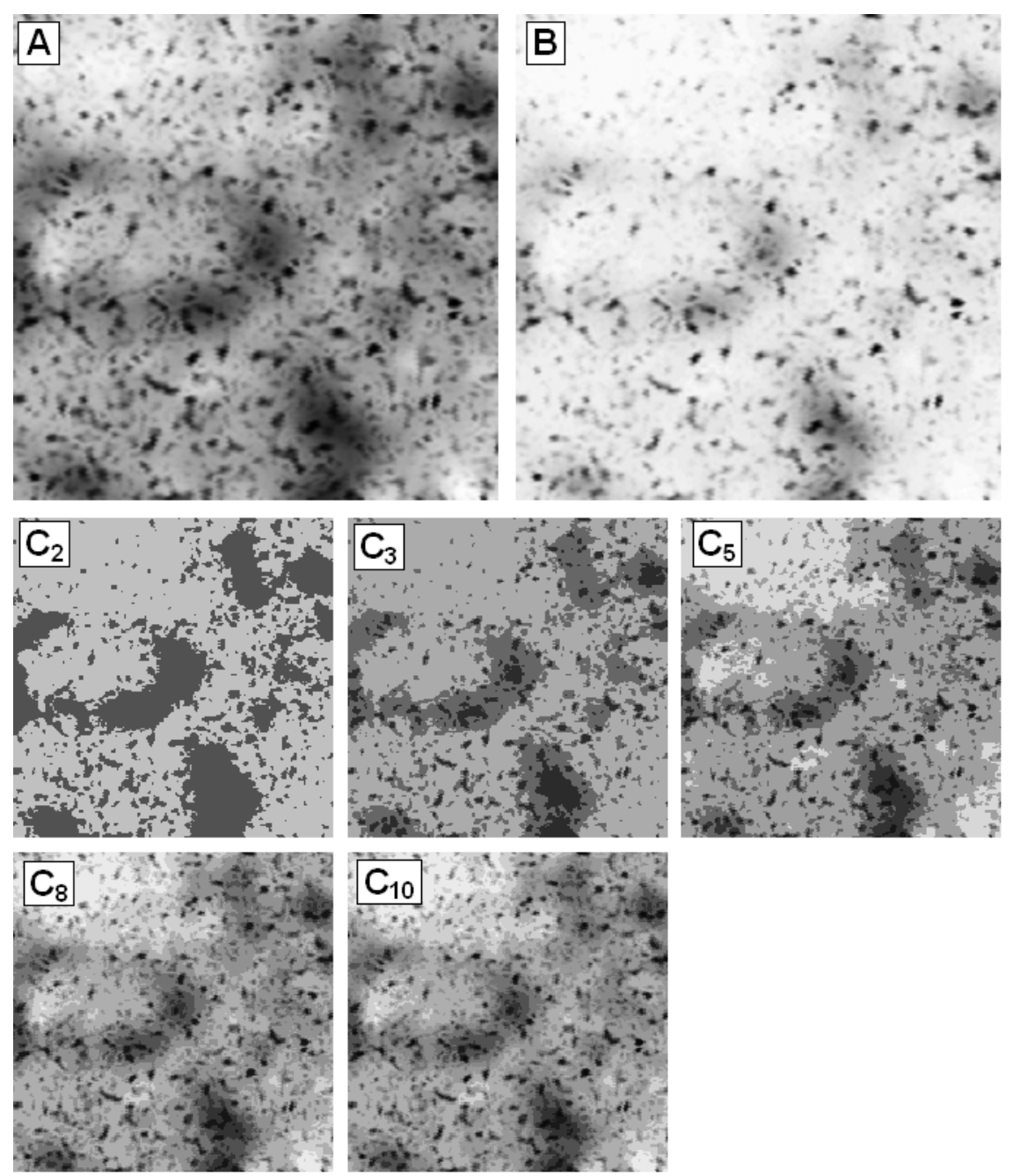

Fig. 2. The virtual landscape used in the simulations. Habitat suitability (A) is assumed to be known to the virtual ecologist, while carrying capacity (B) is unknown. For the analyses, HS was reclassified into $2,3,5,8$, or 10 classes $\left(\mathrm{C}_{2}-\mathrm{C}_{10}\right)$. In panels $A$ and $C$, darker shades represent higher suitability whereas, in panel $\mathrm{B}$, it represents higher carrying capacity.

nal cells. Each cell is characterized by a continuous HS value ranging from 0 to 1 and by a carrying capacity $(K)$ ranging from 1 to 50 individuals. For all subsequent analyses, the HS is known to the virtual ecologist, while carrying capacity is not. I arbitrarily chose an exponential (nonlinear) relationship between HS and $K$, so as to have a patchy landscape (Fig. 2A,B). However, my reasoning would hold for any positive, monotonic 
relationship. The initial abundance of each cell was set to its carrying capacity. Because of regional and demographic stochasticity, abundances varied between 0 and $2 K$, with a tendency to go back to $K$.

At each time step, the population of each cell followed two phases. During the first phase, the population reproduced according to the logistic model:

$$
N_{i}(t+1)=N_{i}(t)+N_{i}(t)\left[r\left(1-\frac{N_{i}(t)}{K_{i}}\right)+\varepsilon_{i}(t)\right]
$$

where $N_{i}(t)$ is population density of the $i$ th cell at time $t ; r$ is the intrinsic growth rate of the population, fixed to $0.3 ; K_{i}$ is the carrying capacity of the $i$ th cell; and $\varepsilon_{i}(t)$ is a coefficient of environmental stochasticity. This stochasticity coefficient is a random deviate obeying a Gaussian distribution with mean 0 and standard deviation either 0.2 (unstable environment) or 0.05 (stable environment). It has a spatial autocorrelation of 0.05 at a distance of 10 cells (following a Gaussian correlogram; see Hirzel et al., 2007). There was no temporal autocorrelation.

After the growth phase, a density-dependent proportion of the population left the cell and distributed itself uniformly among the six adjacent cells. The density dependence followed a sigmoid model. There was always a minimum of $1 \%$ migrants, while the maximum could be either $10 \%$ (philopatric species) or 30\% (disperser species). Alternatively, the species could have density-independent dispersal, with proportions fixed to $10 \%$ or $30 \%$. There could be one, five, or ten subsequent dispersal phases, depending on the species' vagility. Individuals of the most vagile species could thus explore up to 10 cells before the next reproductive phase. A higher number of dispersal phases gives species a better chance of finding the cell with highest suitability and, for density-dependent dispersal, lowest crowding (i.e., IFD). The system ran for 20 generations and the population density of each cell was recorded at the last time step.

In summary, the environment could be stable or unstable, dispersal was either density-dependent or not, the species could be philopatric or a disperser, and there were three levels of dispersal range. In total, I ran 24 different simulations to investigate all combinations of these parameters.

\section{SAMPLING}

As the relative capacity analyses require discrete classes of suitability, the HS map was reclassified into $2,3,5,8$, or 10 classes (Fig. $2 \mathrm{C}_{2}-\mathrm{C}_{10}$ ). Then, a fixed number of cells $(50,100,500$, or 1000) were randomly sampled to estimate population density. As the Maximum and GLM relative capacity analyses only require the HS class and the observed density, they were readily applied to this dataset. However, the isodar analysis requires that cells be grouped by pairs of different suitability combinations. Ideally, cells should be close together and subjected to similar regional stochasticity, i.e., experience the same level of crowding. I tested various distance thresholds for the pairing $(1,2,5$, 10,200 cells). Of course, lower thresholds resulted in lower numbers of pairs. Accordingly, I also recorded the number of pairs for further analysis and the largest distance among the retained pairs. This "random" sampling necessitates no prior knowledge of 
the HS map, which probably corresponds to the most frequent situation. The same data set could thus be used for calibrating the HS model, and then for conducting the relative capacity analysis.

There were 20 replicates of each sampling so as to parry against any sampling effect. To simulate sampling uncertainty, I added four levels of noise to the sampled abundances, i.e., the actual abundance in a cell was multiplied by a random coefficient drawn from a uniform distribution between $-\varepsilon$ and $+\varepsilon$ (where $\varepsilon=0,0.05,0.1$, or 1 ). All in all, for each of the 24 demographic scenarios, reclassified into five numbers of classes, there were five distance thresholds and five sample sizes, replicated 20 times, at four noise levels, totalling 240,000 samples. They were input into the three relative capacity analyses, providing 720,000 analyses. Each method was applied to identical data sets. Moreover, for each of the 240,000 samples, I also drew an ordered set of random relative capacities (drawn from a uniform distribution between 0 and 1) as a control, to provide a baseline against which to compare the three methods.

All analyses were conducted in R (R Development Core Team, 2006) and Tinn-R (Faria et al., 2008).

\section{EVALUATION}

As the relationship between HS and carrying capacity was known, it was possible to compute true relative capacities $F_{i}^{*}(1 \leq i \leq k)$. The results were compared to these values and a mean error coefficient (MEC) calculated as MEC $=\frac{1}{k-1} \sum_{i=1}^{k-1} A b s\left(F_{i}^{*}-F_{1}\right)$. The last class was not included in the computation because it was, by convention, fixed to 1 . This provided four MECs for each of the 240,000 samples (i.e., for three methods and the control).

\section{RESULTS}

Globally, all three methods provided satisfactory assessments of relative capacities. The Isodar method was the most sensitive to noise in the data, in particular at low sample sizes.

Over all samples, median MECs were 0.03 (ranging $=0.0001-0.78$ ) for the Isodar method, 0.05 (from 0.002 to 1.38) for the Maximum method, and 0.04 (0.0001-5.31) for the GLM method, while the median control MEC was $0.27(0.0001-0.75)$. The most crucial parameter was sample size; below five cell pairs, model performance was no better than chance. As the number of cell pairs increased, the median MEC decreased for all three methods, reaching a plateau around 0.05 beyond 50 pairs (Fig. 3A). The proportion of analyses with MEC $<0.1$ increased up to 50 pairs, beyond which Maximum and GLM reached a plateau at $80 \%$ while isodar kept increasing (Fig. 3B).

The three analysis methods produced different results in response to varying number of habitat classes, $k$. For $k=2$, all three methods were roughly equivalent. For $k=3,5$, and 8, the GLM analysis provided better results. However, the GLM was not able to fit the exponential relationship that began to appear at $k=10$ (Fig. 4). The maximum al- 


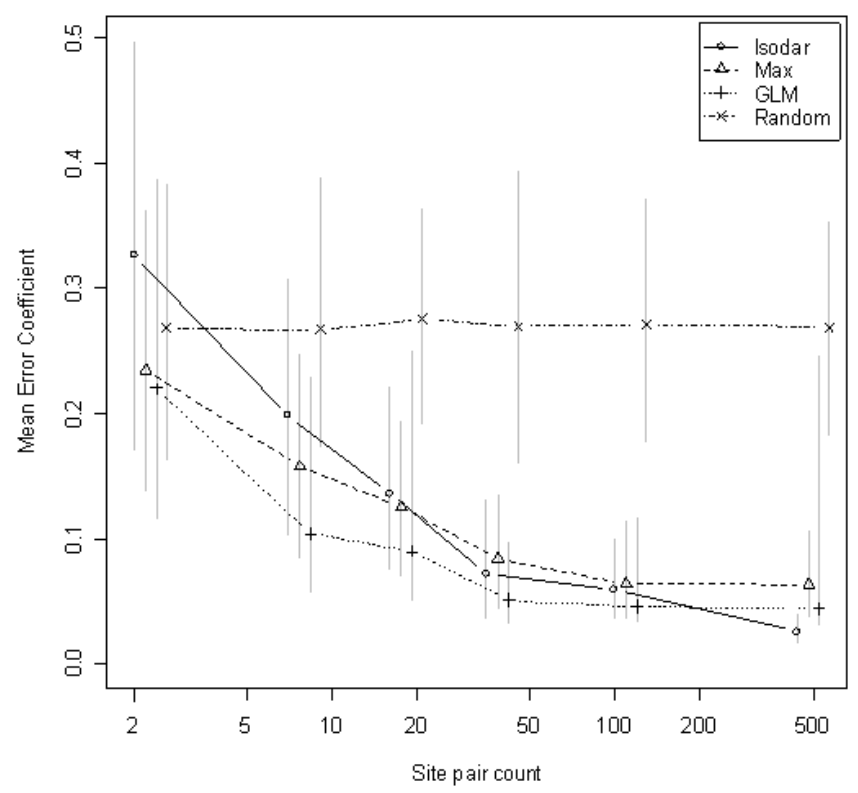

Fig. 3. Effect of sample size on the analysis performances. The main trend line is the median value of the mean error coefficient median, and the interquartile range is represented by gray vertical lines. The horizontal axis is logarithmic.

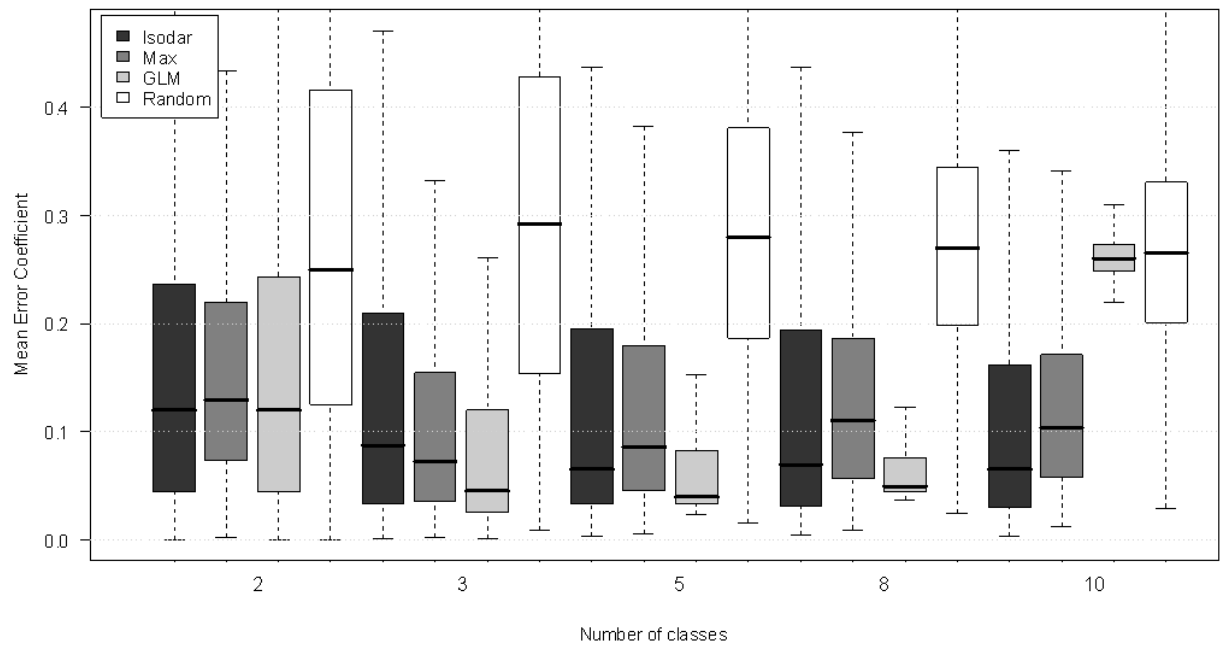

Fig. 4. Distribution of mean error coefficient of the three analyses and control for varying number of predefined habitat suitability classes. The boxes show the interquartile range (IQR) around the black line of the median. The whiskers extend to the most extreme data point, which is no more than 1.5 times the IQR away from the box. 

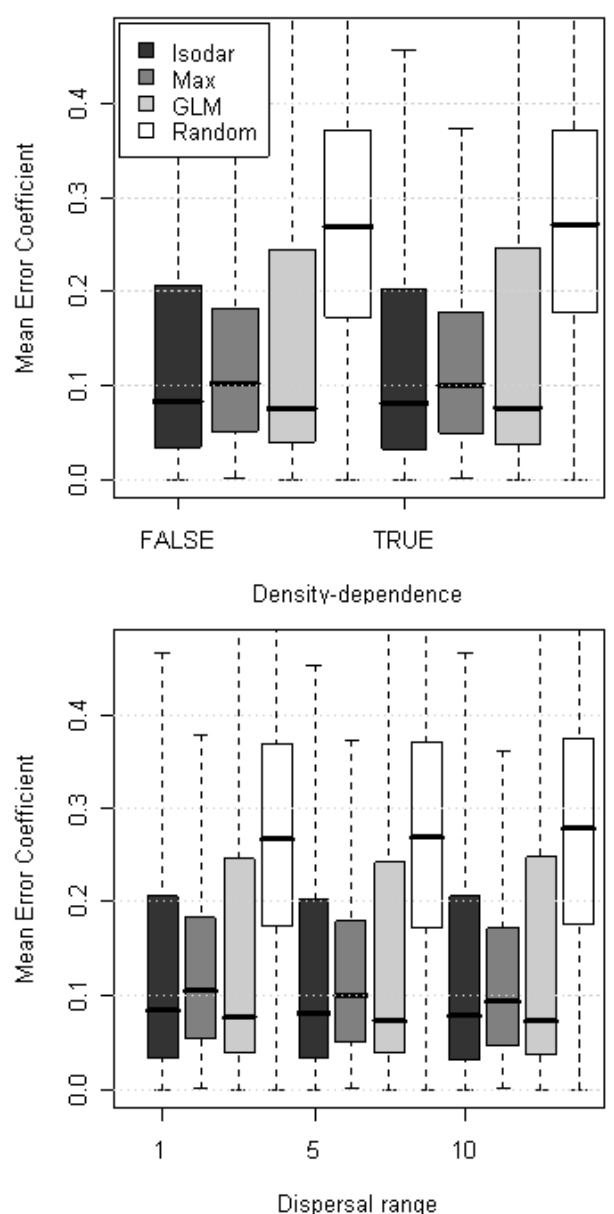
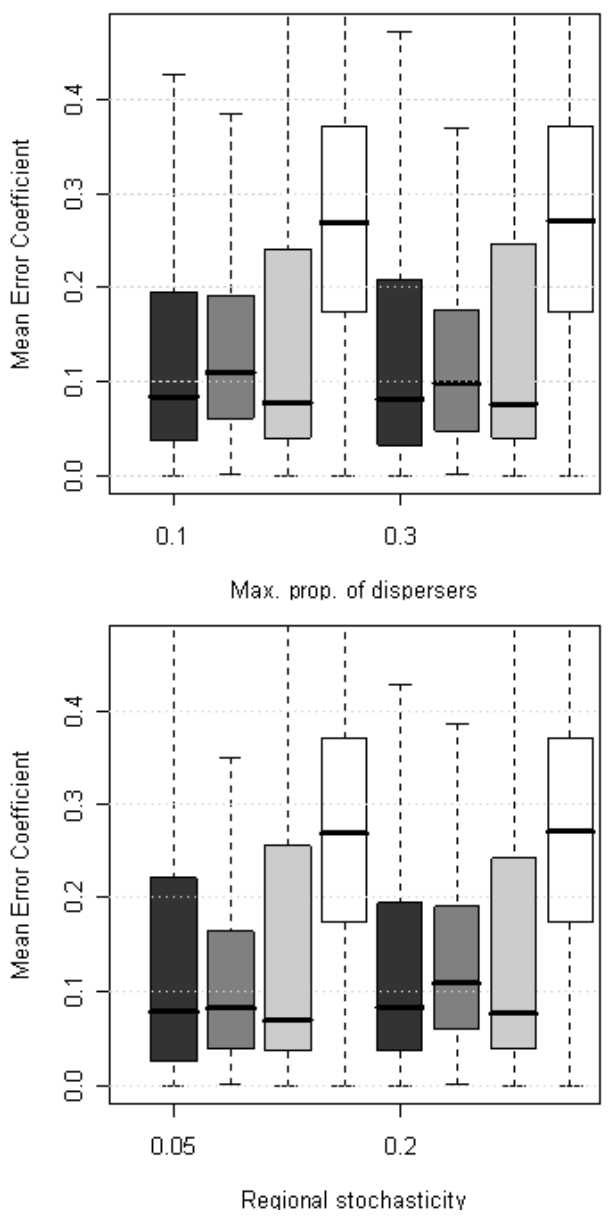

Fig. 5. Box-plots showing the interquartile range (IQR, boxes) around the median (black line) of the mean error coefficients. The panels compare simulations with and without density dependence, and different degrees of dispersal rates, dispersal distances, and regional stochasticity. The whiskers extend to the most extreme data point, which is no more than 1.5 times the IQR away from the box.

lowed distance between sampled cell pairs had no effect per se. With short distances, it was often impossible to get large sample sizes, or to sample all pairs of habitat classes. This in turn affected the results.

The biology of the virtual species had little influence on analysis performance. At low sample sizes, the analyses worked best with disperser rather than philopatric species; however, beyond 50 cell pairs this was no longer important. Density dependence, dispersal distance, and stochasticity also had little effect on results (Fig. 5). 


\section{DISCUSSION}

These simulations showed that it was indeed possible to reliably compute relative capacities for habitat classes by means of density data. As collecting density information is often difficult, it is promising that tolerable results can be reached with measures of density in 5-10 habitat classes. As well, the results showed that 50 samples sufficed to provide almost as good a model as possible. Globally, the three proposed analyses provided equivalent results, with GLM having a slight edge for fewer classes and isodar being better for greater numbers of classes.

When devising the demographic simulations, my expectations were that analyses would depend on the individuals' ability to assess their surrounding environment and crowding and to settle in cells that would provide them with the best fitness. Unexpectedly, none of the parameters I used to influence species' behavior affected analytical results. Even the isodar approach, which is based on the premise that individuals actively select one of several known sites, was not affected. It appears then that the carrying-capacity signal persisted in spite of strong regional stochasticity.

Overall, the methods tended to perform best with a moderate number of habitat classes. This probably stems from the exponential relationship between habitat suitability and carrying capacity. Because classes had equal width on the HS scale, the higher number of habitat classes grouped cells that had a more diverse relative capacity than the lower classes (e.g., compare panels $\mathrm{C}_{2}$ and $\mathrm{C}_{5}$ with panel $\mathrm{B}$ in Fig. 2). Thus, as the number of classes increased, each one grouped more homogeneous cells and thus allowed a higher accuracy to be reached. With real data, the relationships between HS and carrying capacity would not always be linear, which would create the same problem. Therefore, HS classes should be defined carefully. The common practice of defining equal-sized classes should be avoided whenever possible and data-driven reclassification used instead (e.g., see Hirzel et al., 2006; Hirzel and Le Lay, 2008).

The GLM analysis failed with 10 habitat classes. This was due to the quadratic model being unable to fit the exponential HS-carrying capacity relationship. This relationship was masked at low class numbers, likely for reasons suggested above, and it could be sufficiently well approached by linear or parabolic curves (although an increase in the MEC was evident with five and eight classes, Fig. 4). Of course, it would have been possible to fit an exponential curve with the GLM. Indeed, with real data, the actual HScarrying capacity link would be unknown and thus the GLM could suffer from the same problem because it tries to fit a parametric curve to the whole series of densities, while the isodar works on individual pairs of habitats.

In contrast, the Maximum approach takes each habitat class in isolation. This approach appears slightly less accurate than the GLM and the Isodar methods with large numbers of habitat classes. It is too sensitive to extreme events of stochastic density variations. During preliminary analyses, I tested a similar approach that was based on the 90th percentile but it performed in a manner similar to the Maximum method (data not shown).

The GLM and Maximum methods were highly robust to uncertainty in the recorded 
abundances. This is because these methods are based on relative rather than absolute values. Adding noise does not change the relationships statistically and thus had little effect. Only environment-dependent sampling efficiency (e.g., if the species is easier to observe or trap in meadows than in forests) could change the results as it would distort the relationships among the suitability classes. The Isodar method was more sensitive to noise, in particular at low sample size ( $<100$ site pairs), because it is based on the relation between pair abundances. If the noise affects both elements of a pair independently (as it was implemented here) the relationship may be strongly disturbed. In reality, the noise may not be independent. For instance, the two sites of a close pair may have been investigated by the same observer, or under the same sampling conditions. Nevertheless, I would recommend using the Isodar method only with large or noise-free data sets.

Some issues require further investigation. In this study, the HS map was supposed to be perfectly known. However, in real situations, it would suffer from some level of uncertainty. The main effect would be to prevent the use of a high number of habitat classes. With few, wide HS classes (e.g., two to four classes), the uncertainties would be negligible. Therefore, I expect that using real HS maps would not change the outcome predicted by the simulations with low class numbers. Determining how relative capacity is related to carrying capacity and how regional stochasticity affects relative capacity also warrant additional assessment. Finally, it remains to test these analyses on real data, for instance, by comparing populations of flying versus crawling insects.

The relative capacity approach requires two independent surveys. First, a broad-scale survey of species' occurrences over the whole study area provides data needed to build an accurate HS map. Second, this map is used to define a smaller-scale survey of species density in various habitat classes, providing data for the relative capacity analysis. The computation of HS maps on the basis of occurrence data-either presence/absence or presence only - is a mature field, and numerous HS modeling methods have been devised and tested (Guisan and Zimmermann, 2000; Pearce and Boyce, 2006; Peterson, 2006; Soberón, 2007). It is comparatively much easier to collect occurrence data than abundance/density data over broad, heterogeneous areas. Occurrence data could even come from museums, atlases, or online databases (Reutter et al., 2003; Soberón and Peterson, 2004; Gaubert et al., 2006). This approach can be thought of as a hierarchical process: the HS model provides a first assessment, which is then refined by collecting more-focused data. As for the isodar approach, replacing time series by spatial data also allows for shorter studies that could be completed in one or two seasons. An alternative approach would consist of dividing the study area into expert-based habitat classes, and conducting abundance/density surveys in each class. Then, density data could be used in a relative capacity analysis.

Relative capacity may be a useful measure for allowing ecologists to compare quantitatively the demographic value of different habitat classes. Moreover, as it is in effect an areal unit (expressed in $\mathrm{m}^{-2}$ ), it can be added up over all cells of a region to estimate regional relative carrying capacity. Eventually, the third step of the hierarchical process would consist of measuring precisely the absolute areal carrying capacity (measured in individuals $/ \mathrm{m}^{2}$ or territories $/ \mathrm{m}^{2}$ ) of a few habitat classes. If relative capacity is linearly 
related to actual carrying capacity, then it would be theoretically sufficient to compute the proportionality coefficient $(\alpha)$. In practice, it would be safer to measure it over several habitat classes to get several estimations of this coefficient, and to verify the linearity of the relationship.

Relative capacity analyses proposed here provide a promising way of predicting habitat quality using habitat suitability maps and a limited number of focused abundance/density surveys. It should thus provide a valuable tool to field ecologists and wildlife managers. However, to determine their full utility, these approaches should be tested against field data and linked with direct measures of fitness (e.g., survival, reproductive success).

\section{ACKNOWLEDGMENTS}

I wish to thank Gabriel Cisarovsky, Bertrand Fournier, Anaïs Frapsauce, and Loïc Pellissier for creative discussions about the simulations. Comments by Bob Clark, Doug Morris, and two anonymous reviewers greatly improved the clarity of this article. This work was financed by the Swiss National Science Foundation, grant \#3100A0-112511.

\section{REFERENCES}

Austin, M.P., Belbin, L., Meyers, J.A., Doherty, M.D., Luoto, M. 2006. Evaluation of statistical models used for predicting plant species distributions: role of artificial data and theory. Ecol. Model. 199: 197-216.

Begon, M., Harper, J.L., Townsend, C.R. 1996. Ecology. Oxford, Blackwell Science.

Cressman, R., Krivan, V. 2006. Migration dynamics for the ideal free distribution. Am. Nat. 168: 384-397.

Emlen, J. M., Freeman, D. C., Kirchhoff, M. D., Alados, C. L., Escos, J., Duda, J.J. 2003. Fitting population models from field data. Ecol. Model. 162: 119-143.

Faria, J.C., Grosjean, P., Jelihovschi, E. 2008. Tinn-R - GUI/Editor for R language and environment statistical computing. Version 1.19. URL: http://sourceforge.net/projects/tinn-r

Freckleton, R.P., Watkinson, A.R., Green, R.E., Sutherland, W.J. 2006. Census error and the detection of density dependence. J. Anim. Ecol. 75: 837-851.

Fretwell, S.D., Lucas, H.L. 1969. On territorial behavior and other factors influencing habitat distribution in birds. Acta Biotheor. 19: 16-36.

Gaubert, P., Papes, M., Peterson, A.T. 2006. Natural history collections and the conservation of poorly known taxa: ecological niche modeling in central African rainforest genets (Genetta spp.). Biol. Cons. 130: 106-117.

Guisan, A., Thuiller, W. 2005. Predicting species distribution: offering more than simple habitat models. Ecol. Lett. 8: 993-1009.

Guisan, A., Zimmermann, N.E. 2000. Predictive habitat distribution models in ecology. Ecol. Model. 135: 147-186.

Hanski, I. 1991. Single-species metapopulation dynamics: concepts, models and observations. Biol. J. Linn. Soc. 42: 17-38.

Hanski, I. 1998. Metapopulation dynamics. Nature 396: 41-49.

Hirzel, A.H. 2001. When GIS come to life. Linking landscape- and population ecology for large population management modelling: the case of Ibex (Capra ibex) in Switzerland. Ph.D. thesis, University of Lausanne, Lausanne, Switzerland. 
Hirzel, A.H., Le Lay, G. 2008. Habitat suitability modelling and niche theory. J. Appl. Ecol. 45: 1372-1381.

Hirzel, A.H., Helfer, V., Métral, F. 2001. Assessing habitat-suitability models with a virtual species. Ecol. Model. 145: 111-121.

Hirzel, A.H., Le Lay, G., Helfer, V., Randin, C., Guisan, A. 2006. Evaluating the ability of habitat suitability models to predict species presences. Ecol. Model. 199: 142-152.

Hirzel, A.H., Nisbet, R.M., Murdoch, W.W. 2007. Host-parasitoid spatial dynamics in heterogeneous landscapes. Oikos 116: 2082-2096.

Jaquiéry, J., Guélat, J., Broquet, T., Berset-Brändli, L., Pellegrini, E., R. Moresi, Hirzel, A.H., Perrin, N. 2008. Habitat-quality effects on metapopulation dynamics in the greater white-toothed shrew (Crocidura russula). Ecology 89: 2777-2875.

Knight, T.W., Morris, D.W. 1996. How many habitats do landscapes contain? Ecology 77: $1756-1764$.

Krivan, V., Cressman, R., Schneider, C. 2008. The ideal free distribution: a review and synthesis of the game-theoretic perspective. Theor. Pop. Biol. 73: 403-425.

Lin, Y.T.K., Batzli, G.O. 2002. The cost of habitat selection in prairie voles: an empirical assessment using isodar analysis. Evol. Ecol. 16: 387-397.

Manly, B.F., Mcdonald, L.L., Thomas, D.L., Mcdonald, T.L., Erickson, W.P. 2002, Resource selection by animals: statistical design and analysis for field studies. Dordrecht, Kluwer Academic Publishers.

Morris, D.W. 1988. Habitat-dependent population regulation and community structure. Evol. Ecol. 2: 253-269.

Morris, D.W. 1992. Scales and costs of habitat selection in heterogeneous landscapes. Evol. Ecol. 6: 412-432.

Morris, D.W. 2003. Toward an ecological synthesis: a case for habitat selection. Oecologia 136: $1-13$.

Olivier, F., Wotherspoon, S.J. 2005. GIS-based application of resource selection functions to the prediction of snow petrel distribution and abundance in East Antarctica: comparing models at multiple scales. Ecol. Model. 189: 105-129.

Pearce, J.L., Boyce, M.S. 2006. Modelling distribution and abundance with presence-only data. J. Appl. Ecol. 43: 405-412.

Peterson, A.T. 2006. Uses and requirements of ecological niche models and related distributional models. Biodiv. Inf. 3: 59-72.

Potts, J.M., Elith, J. 2006. Comparing species abundance models. Ecol. Model. 199: 153-163.

R Development Core Team. 2006. R: a language and environment for statistical computing, version 2.4.1. R Foundation for Statistical Computing, Vienna, Austria.

Reutter, B.A., Helfer, V., Hirzel, A.H., Vogel, P. 2003. Modelling habitat-suitability using museum collections: an example with three sympatric Apodemus species from the Alps. J. Biogeogr. 30: 581-590.

Shochat, E., Abramsky, Z., Pinshow, B., Whitehouse, M. 2002. Density-dependent habitat selection in migratory passerines during stopover: what causes the deviation from IFD? Evol. Ecol. 16: $469-488$.

Soberón, J. 2007. Grinnellian and Eltonian niches and geographic distributions of species. Ecol. Lett. 10: 1115-1123.

Soberón, J., Peterson, A.T. 2004. Biodiversity informatics: managing and applying primary biodiversity data. Philos. Trans. R. Soc. London Ser. B-Biol. Sci. 359: 689-698.

Soberón, J., Peterson, A.T. 2005. Interpretation of models of fundamental ecological niches and species' distributional areas. Biodiv. Inf. 2: 1-10. 